\title{
Humoral Recognition-Behavioral Stress-Coping Glycolipids Produced By Mice Given Repeated Electroconvulsive Treatment
}

\author{
Yutaka Masuda
}

Psychosomatic Division, Graduate School of Medicine, Akita

${ }^{*}$ Corresponding author

Yutaka Masuda, Psychosomatic Division, Graduate School of Medicine, Akita University, Hondo 1-1-1, Akita 010-8543, Japan

University, Hondo 1-1-1, Akita 010-8543, Japan

Submitted: 20 Apr 2020; Accepted: 25 Apr 2020; Published: 09 May 2020

\begin{abstract}
Background: Stress-coping is a core event of mammalians. Depression symptoms are induced by the stress-coping failures. Repeated electroconvulsive treatment gives a strong stress to mammalians, however, the treatment has been used to improve depression symptoms. Mammalians have recognition-behavioral stress-coping neuronal modulesystem followed by some humoral glycolipids. A sulfated Galbeta1-4GlcNAc-lipid promotes the serotonergic module. GalNAcalpha1-3GalNAc-lipid promotes the adrenergic module. A sulfated Fucalpha1-2Gal-lipid protects the cholinergic module keeping the stress-coping memories from the ischemia-stress. I hypothesized mammalians given repeated electroconvulsive treatment would produce these glycolipids, and would increase the stress-coping ability.
\end{abstract}

Materials and Methods: I examined the glycolipid productions of mice given repeated electroconvulsive treatment under general-anesthesia.

Results: I found mice only given the general-anesthesia produced sulfated Galbeta1-4GlcNAc-lipid and GalNAcalpha13 GalNAc-lipid, and mice given the repeated electroconvulsive treatment under general-anesthesia further produced sulfated Galbeta1-4GlcNAc-lipid and GalNAcalpha1-3GalNAc-lipid, and increased sulfated Fucalpha1-2Gal-lipid production.

Conclusion: Depression symptoms are closely related to serotonergic and adrenergic module activities. I understood repeated electroconvulsive treatment would improve depression symptoms via the sulfated Galbeta1-4GlcNAc-lipid and GalNAcalpha1-3GalNAc-lipid productions.

Keywords: Depression Symptoms, Humoral Glycolipids, Mammalian Stress-Coping System, Neuronal Module Function, Repeated Electroconvulsive Treatment

\section{Introduction}

Depression symptoms are induced by coping a serious stress. Depression patients are very tired, and complain decrease of the physical strength and loss of the eagerness. Repeated electroconvulsive treatment (r-ECT) has successfully decreased the depression symptoms, however, the treatment gives a strong stress for mammalians to show seizures. Many researchers investigated effects of r-ECT on the brain, however, the therapeutic mechanism was not yet clarified. Now, mammalians have recognition-behavioral stress-coping neuronal module-system followed by some humoral glycolipids. I previously reported the stress-coping glycolipids are produced corresponding to quality and quantity of the stressors. A sulfated Galbeta1-4GlcNAc-lipid (sG1-4GN) promoting the serotonergic module is produced to regulate the emotional behaviors for not-wasting the physical strength, GalNAcalpha1-3GalNAc-lipid (GN1-3GN) promoting the adrenergic module is produced to induce the stress-coping behaviors, and sulfated Fucalpha1-2Gal-lipid $(\mathrm{sF} 1-2 \mathrm{G})$ is produced to protect the cholinergic module keeping the stress-coping memories from the ischemia-stress [1]. I reported the recognition-behavioral stress-coping glycolipid-production system works in human [2]. So, I hypothesized r-ECT would produce the stress-coping glycolipids via mammalian stress-coping system, and the glycolipids would induce the anti-depression effects. Recently, r-ECT under general-anesthesia has been performed as a therapeutic method for depression patients, so, I investigate the humoral stresscoping glycolipid productions of mice given r-ECT under generalanesthesia in the present study.

\section{Materials and Methods}

Animal

Female 9 weeks-old DDY mice were purchased from Japan SLC Co. (Hamamatsu, Japan) for using in the present study. All of the experiments were conditioned in accordance with Animal Research Regulations of Akita University School of Medicine (the approval number: a-1-3019). 
Treatmented Mice

Isoflurane General-Anesthesia and Electroconvulsive Treatment

For general-anesthetizing, mice were individually placed in a glass container holding $500 \mathrm{ml}$ filled with vapor of isoflurane (FujiFilm and Wako-Junyaku Co. Osaka, Japan) for 10s. Sine-wave electric current $(100 \mathrm{~V}, 50$ cycles per min) was given to head of the anesthetized mice via electroconvulsive therapy apparatus (Sakai Co., Tokyo, Japan) for 3s. Validity of the electroconvulsive treatment was assessed with mice tonic convulsion.

\section{Control Mice}

A 6 mice were individually placed in the container without isoflurane vapor for 10s 6times per 2 days. They were considered as Control mice. They were sacrificed by the neck-dislocation, and their blood was collected $24 \mathrm{~h}$ after the final treatment. The serum was pooled and restored at $4{ }^{\circ} \mathrm{C}$.

\section{Mice Given General-Anesthesia}

A 6 mice were individually given the general-anesthesia 6times per 2 days. They were considered as ANZ mice. They were sacrificed by the neck-dislocation, and their blood was collected $24 \mathrm{~h}$ after the final treatment. The serum was pooled and restored at $4{ }^{\circ} \mathrm{C}$.

\section{Mice Given Repeated Electroconvulsive Treatment}

A 6 mice were individually given the electroconvulsive treatment under the general-anesthesia 6times per 2 days. They were considered as r-ECT mice. They were sacrificed by the neck-dislocation, and their blood was collected $24 \mathrm{~h}$ after the final treatment. The serum was pooled and restored at $4{ }^{\circ} \mathrm{C}$.

\section{Humoral Glycolipid Fractionation}

Glycolipids are variously charged in water. Fractionation of humoral glycolipids was performed as previous described [1]. Briefly, $2.5 \mathrm{ml}$ of chloroform and $5 \mathrm{ml}$ of methanol were added to $2 \mathrm{ml}$ of the pooled serum. The solution was intensively mixed for $3 \mathrm{~min}$ and incubated for $10 \mathrm{~min}$ at room temperature (RT). Then, $2.5 \mathrm{ml}$ of chloroform was added to the solution, followed by intensive mixing for $30 \mathrm{~s}$. A $2 \mathrm{ml}$ of water was added to the solution, followed by intensive mixing for another $30 \mathrm{~s}$. The mixture was then centrifuged at $150 \times \mathrm{g}$ for $10 \mathrm{~min}$ at RT. The chloroform (lower) layer was collected, and the solvent was evaporated at RT. The extracted lipids were then suspended in $2 \mathrm{ml}$ of water. The solution was applied to $2 \mathrm{ml}$ of anion-exchanger DE-52 (Whatman Co., Maidstone, UK) column, which had been saturated with $10 \mathrm{mMNaHCO} 3, \mathrm{pH} 8.3$ and washed with water. The lipids were eluted with $1 \mathrm{ml}$ consecutive washes of $50.100,150,200,250$ and $300 \mathrm{mMNaCl}$. Fractions eluted with 100,150 and $250 \mathrm{mMNaCl}$ were then diluted to $2 \mathrm{ml}$ with water as the present samples.

\section{Sulfate-Radical Eliminating Method}

Humoral stress-coping glycolipid fractionated with 100 or $250 \mathrm{mMNaCl}$ is sulfated. Sulfate-radical of the glycolipids disturbs the terminal sugar-chain reactivity detection. Sulfate-radical of the recognition-behavioral stress-coping glycolipids was eliminated as previously described [1]. Briefly, glycolipids were re-extracted from $800 \mu 1$ of the sample solutions using methanol and chloroform as described above. The extracted glycolipids were added $400 \mu 1$ of the reagent containing silyl-agents and dehydrated pyridine of TMS-HT kit (Tokyo Chemical Industry Co., Tokyo, Japan), and then, were incubated at $90{ }^{\circ} \mathrm{C}$ for $3 \mathrm{~h}$. The solutions were added $800 \mu \mathrm{l}$ water, intensively mixed for $30 \mathrm{~s}$ and were restored in $4^{\circ} \mathrm{C}$ for $24 \mathrm{~h}$.

\section{Measurement of the Glycolipid Production}

Glycolipids are bipolar, and they attach to plastic plate in 50\% ethanol condition. A modified Enzyme-Linked ImmunoSorbent Assay (ELISA) was performed for measuring the glycolipids production as previously described [1]. Briefly, the sample solution eluted with $150 \mathrm{mMNaCl}$, the sulfate-radical-eliminated sample solution fractionated with 100 or $250 \mathrm{mMNaCl}$, or physiological saline (PS) as a blank control, were prepared to $50 \%$ ethanol solution. A $100 \mu 1$ of the solution was poured into a well of a 96-well plastic plate (Sumitomo-Bakelite Co., Tokyo, Japan). The ELISA was performed with the use of $300 \mu \mathrm{l}$ of $5 \%$ bovine serum albumin (Sigma-Aldrich Co., St. Louis, MO, USA) as a blocker, a biotinylated lectin of ricinus communis recognizing Galbeta1-4GlcNAc, that of Dolichos biflorus recognizing GalNAcalpha1-3GalNAc or that of aleuria aurantia recognizing Fucalpha1-2Gal, peroxidase-conjugated-avidin (Seikagaku Co., Tokyo, Japan), and the coloring kit (Sumitomo Bakelite Co.). Then, the light absorbance was measured at the dual wavelength of $450 / 655 \mathrm{~nm}$. The ELISA procedure was individually performed on different 5 plates.

Statistical Analyses

Steel-Dwass test was used for finding statistical difference. A $p<0.05$ was considered as a significant difference.

Results

A Sg1-4gn Production

A sG1-4GN is produced in fraction eluted with $100 \mathrm{mMNaCl}$. The production was detected in all of the samples. The ANZ mice produced the glycolipid more than the Control mice did, and the r-ECT mice produced more than the ANZ mice did [Table 1].

Table 1: Mean \pm Sd of Sg1-4gn Reactivity in the Samples

\begin{tabular}{|l|c|}
\hline Sample obtained from & Light absorbance (450/655 $\mathbf{~ m})$ \\
\hline Control mice (not-treated) & $0.119 \pm 0.005$ \\
\hline ANZ mice (Anesthetized) & ${ }^{*} 0.179 \pm 0.012$ \\
\hline r-ECT mice (Given repeated ECT) & ${ }^{*}: 0.211 \pm 0.011$ \\
\hline Blank (Physiological saline) & $0.054 \pm 0.008$ \\
\hline
\end{tabular}

A sG1-4GN: sulfated Galbeta1-4GlcNAc-lipid promoting the serotonergic

module.

$\mathrm{p}<0.05$ compared to Control mice (Steel-Dwass test)

$\# \mathrm{p}<0.05$ compared to ANZ mice (Steel-Dwass test)

ECT: electroconvulsive treatment

\section{GN1-3GN production}

GN1-3GN is produced in fraction eluted with $150 \mathrm{mMNaCl}$. The production was detected in all of the samples. The ANZ mice produced the glycolipid more than the Control mice, and the r-ECT mice produced more than the ANZ mice did (Table 2). 
Table 2: Mean \pm SD of GN1-3GN reactivity in the samples

\begin{tabular}{|l|c|}
\hline Sample obtained from & Light absorbance (450/655 $\mathbf{~ m m})$ \\
\hline Control mice (not-treated) & $0.066 \pm 0.005$ \\
\hline ANZ mice (Anesthetized) & $\approx 0.113 \pm 0.021$ \\
\hline r-ECT mice (Given repeated ECT) & $\# \approx 0.189 \pm 0.036$ \\
\hline Blank (Physiological saline) & $0.049 \pm 0.004$ \\
\hline
\end{tabular}

GN1-3GN: GalNAcalpha1-3GalNAc-lipid promoting the adrenergic module.

$\approx \mathrm{p}<0.05$ compared to Control mice (Steel-Dwass test)

$\# \mathrm{p}<0.05$ compared to ANZ mice (Steel-Dwass test)

ECT: electroconvulsive treatment

A sf1-2g Production

A $\mathrm{sF} 1-2 \mathrm{G}$ is produced in fraction eluted with $250 \mathrm{mMNaCl}$. The production was detected in all of the samples. The r-ECT mice produced the glycolipid more than the ANZ mice and the Control mice did [Table 3].

Table 3: Mean \pm Sd of Sf1-2g Reactivity in the Samples

\begin{tabular}{|l|c|}
\hline Sample obtained from & Light absorbance (450/655 $\mathbf{~ m})$ \\
\hline Control mice (not-treated) & $0.115 \pm 0.014$ \\
\hline ANZ mice (Anesthetized) & $\approx 0.136 \pm 0.010$ \\
\hline r-ECT mice (Given repeated ECT) & $\# \approx 0.137 \pm 0.006$ \\
\hline Blank (Physiological saline) & $0.045 \pm 0.006$ \\
\hline
\end{tabular}

A sF1-2G: sulfated Fucalpha1-2Gal-lipid protecting the cholinergic module.

$\approx \mathrm{p}<0.05$ compared to Control mice (Steel-Dwass test)

ECT: electroconvulsive treatment

\section{Supplementary Data}

Raw data of light absorbance indicating the glycolipid production

Light absorbance $(450 / 655 \mathrm{~nm})$

\begin{tabular}{|l|c|c|c|c|c|}
\hline \multicolumn{2}{|l|}{ Plate } & $\mathbf{2}$ & $\mathbf{3}$ & $\mathbf{4}$ & $\mathbf{5}$ \\
\hline sG1-4GN & \multicolumn{5}{|c|}{$\mathbf{2}$} \\
\hline Control & 0.111 & 0.119 & 0.126 & 0.123 & 0.120 \\
\hline ANZ & 0.162 & 0.171 & 0.179 & 0.189 & 0.197 \\
\hline r-ECT & 0.220 & 0.214 & 0.207 & 0.208 & 0.209 \\
\hline Blank (PS) & 0.057 & 0.061 & 0.065 & 0.054 & 0.043 \\
\hline & & GN1-3GN & & & 0.067 \\
\hline Control & 0.055 & 0.061 & 0.077 & 0.072 & 0.111 \\
\hline ANZ & 0.149 & 0.119 & 0.089 & 0.100 & 0.229 \\
\hline r-ECT & 0.165 & 0.172 & 0.178 & 0.203 & 0.054 \\
\hline Blank (PS) & 0.052 & 0.048 & 0.043 & 0.049 & 0.205 \\
\hline & & & & & 0.194 \\
\hline Control & 0.205 & 0.199 & 0.193 & 0.221 & 0.253 \\
\hline ANZ & 0.196 & 0.203 & 0.210 & 0.252 & 0.061 \\
\hline r-ECT & 0.235 & 0.244 & 0.252 & 0.050 & \\
\hline Blank (PS) & 0.057 & 0.099 & 0.040 & & \\
\hline
\end{tabular}

\section{Discussion}

Since it was recognized human seizures have a therapeutic potential to the psychotic symptoms, r-ECT was considered as a therapeutic method to treat the psychotic symptoms, and has been established as a method decreasing the severe depression symptoms [3]. Antidepressive effects of r-ECT has been variously investigated [4-6], however, any investigations did not clarified the anti-depression mechanism.

Depression patients generally complain decrease of the physical strength and loss of the eagerness. This indicates depression patients have the serotonergic module dysfunction and the adrenergic module dysfunction in their stress-coping system. In fact, antidepressants improve depression symptoms by stimulating the serotonergic neuronal activity and the adrenergic neuronal activity. Now, sG1$4 \mathrm{GN}$ is produced to promote the serotonergic module, GN1-3GN is produced to promote the adrenergic module, and a $\mathrm{sF} 1-2 \mathrm{G}$ is produced to protect the cholinergic module from the ischemia- stress [1]. In the present study, the Control mice produced sG14GN, GN1-3GN and sF1-2G. This suggests mice always prepares these glycolipids for the stress-coping. The ANZ mice produced sG1-4GN and GN1-3GN more than the Control mice did. This suggests the mice recognized the general-anesthesia as a stress to be coped with the serotonergic module activity and the adrenergic module activity. In fact, depression patients often complain insomnia, and improvement of the sleep-disturbance deceases the depression symptoms. The r-ECT mice produced sG1-2GN and GN1-3GN more than the ANZ mice did, and increased sF1-2G production. These suggest the mice recognized r-ECT not only as a stronger stress to be coped with the serotonergic module activity and the adrenergic module activity but also as an ischemia-stress decreasing the cholinergic module activity. Amnesia observed after r-ECT might be induced via ischemia-stress of the cholinergic module.

A strong stress induces secretion of gene-expressing hormones via Hypothalamus-Pituitary Axis. T3 and TSH levels of major 
depression patients are lower than those of healthy controls [7], and r-ECT increases a gene-transcription [8]. Mice and depression patients given r-ECT might increase the stress-coping glycolipidgene transcriptions via the hormones secretion. Nevertheless, mechanism of the glycolipids produced in peripheral blood is not yet clear in the present study.

\section{Conclusion}

Depression symptoms are closely related to serotonergic and adrenergic module activities. I understood repeated electroconvulsive treatment would improve depression symptoms via the sulfated Galbeta1-4GlcNAc-lipid and GalNAcalpha1-3GalNAc-lipid productions.

\section{Ethical Approval}

All of the presented experiments were conditioned in accordance with Animal Research Regulation of Akita University School of Medicine (the approval number: a-1-3019).

Funding Information

The present study was performed without financial supports.
References

1. Masuda Y, Narita H, Hasegawa H. (2019) Stress-coping humoral glycolipids produced by mice given controlled bathing treatments. Hindawi Neuroscience Journal

2. Masuda Y. (2020) A humoral recognition-behavioral stresscoping glycolipid considered as another biomarker of psychotic symptoms of schizophrenia. Advances in Neurology and Neurosciences. 3: p37-40.

3. Qiu H, Li X, Zhao W, Du L, Huang P, et al (2016) Electroconvulsive Therapy-induced Brain Structural and Functional Changes in Major Depressive Disorders. Med. Sci. Monit 22: 4577-4586.

4. Tsen P, Mansari M, Blier P (2013) Effects of repeated electroconvulsive shocks on catecholamine systems: electrophysiological studies in rat brain. Synapse 67: 716-727.

5. Yrondi Y, Peran P, Sauvaget A, Schmitt L, Arbus C (2018) Structural functional brain changes in depressed patients during and after electroconvulsive therapy. Acta Neuropsychiatr 30: p17-28.

6. Huang G, Chen CH. (2008) Identification of gene transcript in rat frontal cortex that are regulated by repeated electroconvulsive seizure treatment. Neuropsychopharmacology 58: 171-177.

7. Stipcevic T, Pivac N, Kozaric-Kovacic D, Muck-Seler D (2008) Thyroid activity in patients with major depression. Coll. Antropol 32: 973-976. 